\title{
GOM: New Genetic Optimizing Model for Broadcasting Tree in MANET
}

\author{
Said Elaiwat ${ }^{1}$, Ammar Alazab $^{1}$, Sitalakshmi Venkatraman ${ }^{2}$ Mamoun Alazab $^{2}$ \\ ${ }^{1}$ School of Information Technology \\ Deakin University, Australia \\ \{ selaiwat, aalazab\}@deakin.edu.au \\ ${ }^{2}$ Graduate School of ITMS \\ University of Ballarat, Australia \\ \{s.venkatraman, m.alazab\}@ballarat.edu.au
}

\begin{abstract}
Data broadcasting in a mobile ad-hoc network (MANET) is the main method of information dissemination in many applications, in particular for sending critical information to all hosts. Finding an optimal broadcast tree in such networks is a challenging task due to the broadcast storm problem. The aim of this work is to propose a new genetic model using a fitness function with the primary goal of finding an optimal broadcast tree. Our new method, called Genetic Optimisation Model (GOM) alleviates the broadcast storm problem to a great extent as the experimental simulations result in efficient broadcast tree with minimal flood and minimal hops. The result of this model also shows that it has the ability to give different optimal solutions according to the nature of the network.
\end{abstract}

Keywords-MANET; Network; Genetic Algorithm; Optimisatoin.

\section{INTRODUCTION}

Broadcasting is the process in which a source node sends a message simultaneously to all other nodes in the network. Data broadcasting in a mobile ad-hoc network (MANET) is a common method of information dissemination in many applications, such as, for sending critical information to all hosts, for signalling an alarm or for paging all host [1] and for routing packets in several network routing protocols [2]. Furthermore, the inherent nature of MANET is quite different from wired networks in terms of its multi-hop structure, mobility and broadcasting process due to the nonavailability of wired link among hosts. When each host sends a particular message to all its neighbors within a radius range, broadcast storm problem arises due to its high density.

The broadcast storm problem arises due to three main reasons, namely redundancy, contention, and collision [3]. Redundancy happens when a mobile host decides to retransmit the message to its neighbours when its neighbors have already received this message. Contention takes place when a mobile host transmits a message to its neighbours and simultaneously the neighbors decide to retransmit the message which may cause nodes to strongly contend for network access. Collision is said to happen when two nodes simultaneously try to transmit messages between nodes each other [3]. Since all these three are broadcasting storm problems lead to many redundant messages being transmitted, it results in inefficiencies. Hence, our goal in this research study is to propose a model for reducing retransmit messages in the network for dynamically changing MANETs. However, this work shows that the key of decreasing retransmit messages will decrease to almost remove broadcast storm problem redundancy, contention, and collision.

The organisation of this paper is as follows. In Section II we provide a brief background and survey of literature related to the study. We classify the commonly used broadcasting techniques in Section III and describe the minimum spanning tree approach in Section IV. In Section $\mathrm{V}$ we propose an innovative Genetic Optimisation Model and explain its implementation details and efficiency in Section VI. We project the results of our experimental simulation and a comparative study in Section VII. Finally, in Section VIII we provide the conclusions of this study and future work.

\section{BACKGROUND AND RELATED LITERATURE}

Many techniques are proposed to optimize the broadcasting process in a MANET. Traditionally, simple flooding [4] is used as a straight-forward technique as it does not require any complex computations. Since it is easy to implement and has high reliability, it is a commonly adopted method to date. However, this technique harms the resources of the network because it suffers from the serious Broadcast Storm Problem due to huge amounts of retransmitted messages. Nevertheless, it is still suited for mobile networks, especially when the network has mobility, and also in some situations when the reliability is the main goal at the expense of anything like the battlefield survivability [5], [6].

Many researches have proposed different techniques for optimizing the broadcast process. A method called 'Geoflood' [7] aims to optimize the flooding process by refraining from retransmitting the message when the message has been received from some other node. Tseng et al. [3] have proposed five different methods, namely, Probabilistic Scheme, Counter Based Scheme, Distance Based Scheme, Location Based Scheme and Cluster Based Scheme to solve the Broadcast Storm Problem. On the other hand, some researchers studied the complexity of finding minimum flood tree that has minimum hops and Lipman et 
al. [8] have proposed using Localized Minimum Spanning Tree Flooding (LMSTFlood) to reduce duplicate message reception, consumption of energy and average transmission distance. However, most of these do not cater to different optimal solutions with the dynamically changing MANET structure, which is our goal here.

\section{ClassificAtion OF BROAdCASTING TECHNIQUES}

Many algorithms try to optimize the broadcasting process and to minimize the broadcast storm problem. The most commonly used broadcasting techniques are classified below.

\section{A. Simple Flooding}

As explained in previous section, this technique is suited for low node densities in MANET and high mobility as computations are simple [5], [6], but it causes broadcast storm problem in high proportions. Also, it is important for some other situations such as reachability. However, we have implemented this method to have a comparative study as against the performance of our proposed genetic model.

\section{B. Threshold Based Schemes}

In these schemes, thresholds are based on probability such as Simple Probabilistic Based Broadcasting (SPBB), or counters called Counter Based Scheme (CBS), or distances calculated, namely Distance Based Broadcasting Technique (DBB), or the location of the mobile node, called Location Based Broadcasting (LBB) [3].

\section{Cluster Based Broadcasting}

This method depends on dividing the area of the network into sub areas called cluster, each cluster consists of hosts, cluster head and gateway [3].

\section{Topology Based Techniques}

Some techniques are based on determining the network topology and eliminating neighbours [5]. In a typical MANET topology, this is dynamically changing

\section{E. Neighbor Knowledge Technique}

The notion from this technique is reducing the number of forwarded nodes (relay nodes) to avoid the problems resulting from flooding. This technique finds the smallest set of forwarded nodes, after covering all nodes in the network, (nodes either in the set or the neighbor of nodes in the set), and the smallest set is called a Connected Dominating Set (CDS), especially applicable in routing [4].

\section{IV.SPANNING TREE BROAdCASTING TECHNIQUE (STB)}

The manner in which MANET works is totally different from other networks. When any host broadcasts a message to all its neighbors, all nodes within its radius will receive the message. Therefore, with the absence of wired link among hosts, computing cost depends on the number of forward nodes (number of hops) that means we need to find minimum hops tree. On the other hand, the number of redundant messages must be minimized (minimize or remove broadcast storm problem) and overhead in bandwidth is reduced. This leads us to find minimum flood tree.

Finding Minimum Spanning Tree (MST) [9] in normal state does not need complex computations and can be solved by any known algorithm such as, Prim's algorithm [10]. However, special type of spanning trees tries to optimize two or more parameters at the same time, like the k-hop minimum spanning trees (K-MST) [11]. Eppstein has provided a simple summary about the main types of spanning trees and the complexity of each of them [12].

\section{GeNETIC OPTIMISATION MODEL (GOM)}

While some studies have used genetic algorithm (GA) for multicast problem [13] or to minimise wireless power problem [14], [15] the purpose of this research study is to propose an efficient GA model that has the ability to find a minimal flood tree with minimal hops. A configured mobile ad-hoc network (MANET) is the input of the model and the broadcast tree is its output. The proposed GA when applied on a graph

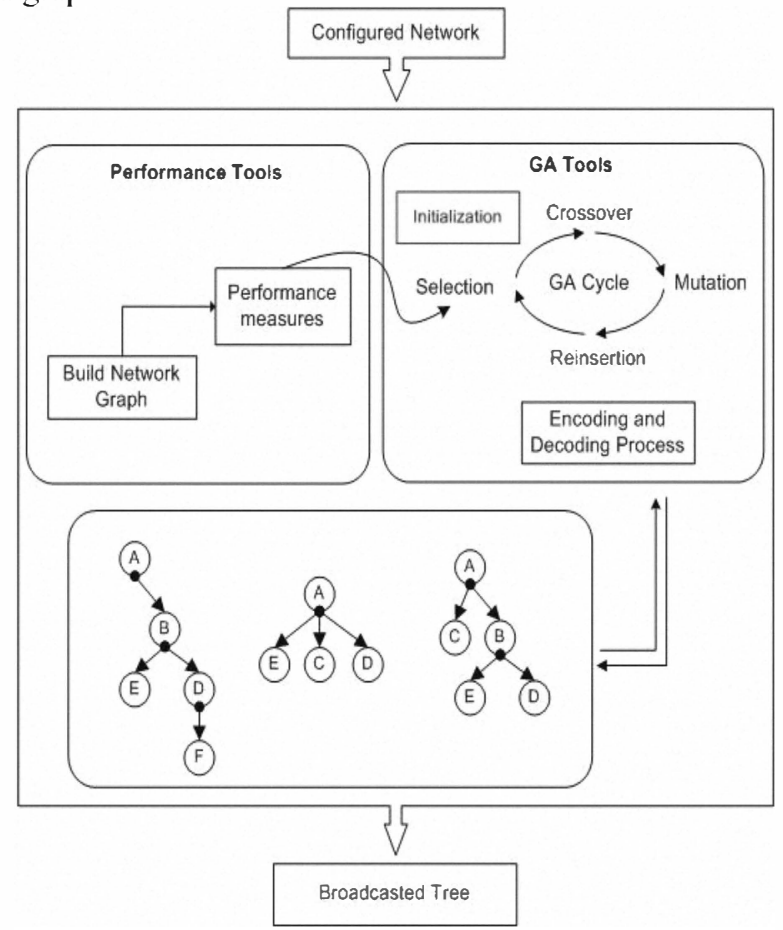

Figure 1 Proposed Genetic Optimisation Model (GOM)

represented MANET produces the optimal broadcast tree with many different solutions that is unique from other studies [16].

The GOM components (Figure 1) consist of Performance Tools to measure the performance of the network and the GA Tools to evolve with the structure of the networks. We represent the source tree (phenotype) as a 
graph and make use of matrix representation (genotype) as shown in Figure 2 with

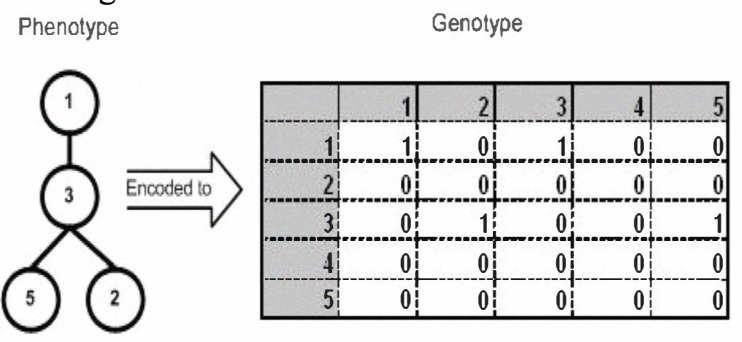

Figure 2 Phenotype and Genotype representation certain rules to reduce the gap between genotypic and phenotypic space as much as possible. Now, genes here are the cells in the matrix, where row number represents the node (parent node) while column number represents children of parent node, The intersection between row and column gives a cell representing the link between parent and child. If there is a network connection between the two nodes, then the value of the cell will be 1, otherwise 0 . Each row that has 1 's is a parent node, leaf nodes are rows without 1 's and disconnected node is a node without 1 's in rows and columns. Root node is a row that has one along the diagonal of the matrix.

\section{IMPLEMENTATION OF GOM}

The genetic algorithm (GA) inspired by natural evolution generates solutions to optimization problems in a way to search heuristics that mimics the process of natural evolution operations such as selection, crossover, mutation and insertion.

\section{A. Selection}

Among the different types of selection, we will use Roulette Wheel Selection in our model; which is a simple technique and gives the needed cover for demand of our system. It works as individuals are given a probability of being selected that is directly proportionate to their fitness. Two individuals are then chosen randomly based on these probabilities and produce offspring.

\section{B. Modification}

Modification consists of two parts: crossover and mutation. The result of modification (crossover \& mutation) must be verified as a correct tree according to the topology of the network.

Crossover: Crossover operation, between two parents, must be between nodes of the same name, and at any part of tree. Figure 3 illustrates crossover operation between P1 and P2, A sub tree from P1 and a sub tree from P2 are chosen to be cut randomly such that the crossover point is the same node in two parents. For example, Node "A" in the child 1 cannot see node "D" (out of range node "A") and therefore the link between node "A" and node " $\mathrm{D}$ " is not correct. Also, node "B" in child2 cannot see node "C" (out of range of node "B"). Hence, we have designed the rule that crossover point

Figure 3 Crossover in Same Node in the Parents (node B).

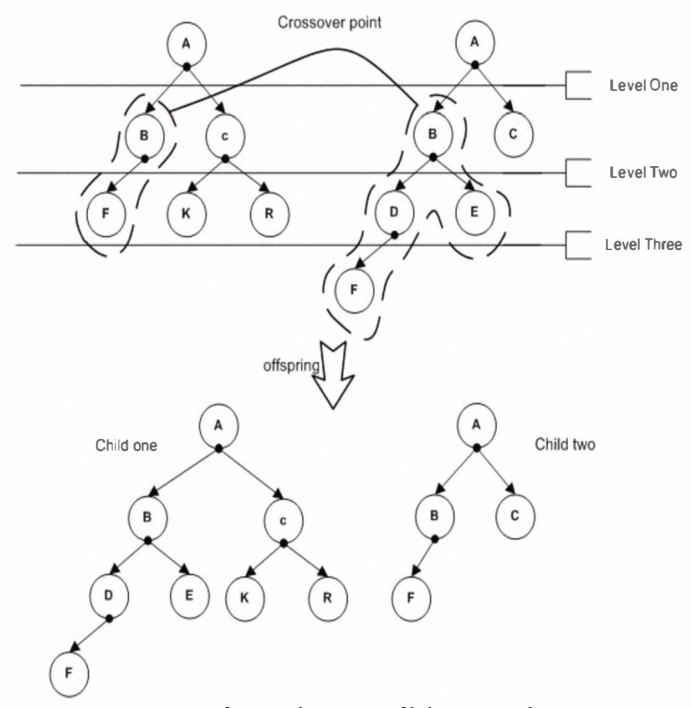

between two parents is only possible on the same node name in parents so as to avoid Out of Range Problem. Similarly, we have devised a rule to avoid Redundant Nodes Problem when some nodes may become redundant in a tree after crossover. To avoid this problem, we proposed the following steps: first we have to remove one of these nodes according to the type of node, if it is leaf node with parent node under the same the same we select leaf node to remove. othercase, if leaf node with another leaf node we select the higher level same thing if we have parent node with another parent node.. To reflect this on matrix, we perform the following three steps: 1) make crossover in the same row number between two trees (individuals); 2) in each tree, find a set of children and descendants that the chosen row (crossover point) is a parent of; and 3) finally interchange parts between trees. Sometimes, we face a problem in one of children - there maybe redundancy among nodes (twice 1's are present in the same column). In such a situation, we need to reset the leaf node to zero (remove leaf node from the tree).

Mutation: After selection and crossover, we now have new individuals (trees). However, to ensure that the individuals are not exactly the same, we make small changes (probability) by using mutation. We begin by looping through all the nodes of all individuals (genes), and if the gene is selected according to the ratio for mutation, we can either change it by a small amount or replace it with a new value. We allow the probability of mutation to reach up to $7 \%$ (based on prior trials). In this study, we have divided mutation operation into two parts: 1) mutation in parent node, and 2) mutation in leaf node. We have designed rules depicting reality such that mutation in the 
parent node can be done only by adding new child to the parent, while mutation in the leaf node can be done by adding new child to the leaf node (becomes now a parent node) or by deleting the leaf node. Before make a mutation operation, we verify two aspects: 1) this node must be disconnected (cell value set to 0 ); and 2) adding nodes must adhere to the network topology.

\section{Reinsertion}

Once the offspring have been produced or generated by selection, crossover and mutation of individuals from the old population (parents), we need a mechanism to replace or to combine the old generation with the new generation, the population size here is fixed and it cannot be increased, Therefore, the new population contains the best individuals according to the best values of their fitness. We have designed the fitness function to depend on two parameters, number of hops and redundancy. We also check if less offspring are produced than the size of the original population and in order to maintain the size of the original population, the offspring may have to be reinserted into the old population. Similarly, we have introduced a reinsertion scheme to determine if not all offspring are required to be used at each generation so that offspring generated do not exceed the size of old population.

\section{EXPERIMENTAL RESULTS}

We have implemented the proposed GOM simulator using Java NetBeans IDE5.5. As shown in Figure 4, the simulator accepts two sets of inputs: 1) environmental parameters such as, number of nodes, their radius, and more importantly the mobility factor for the network, and 2) broadcasting parameters such as, type of broadcasting techniques to be used and the required broadcasting variables.

We have performed 50 simulation runs and then the results were averaged. The comparison between the two approaches: simple flooding and GOM was done based on two performance metrics, number of hops and redundancy. Studies have indicated that simple flooding performs well in number of hops. In our experiment, simple flooding and our GOM model gave similar results and only in some occasions GOM takes slightly higher number of hops than simple flooding, which does not care about redundancy. Below, (1) gives the formula of total number of hops, NHops, where $H$ is the hops in the network calculated during each simulation.

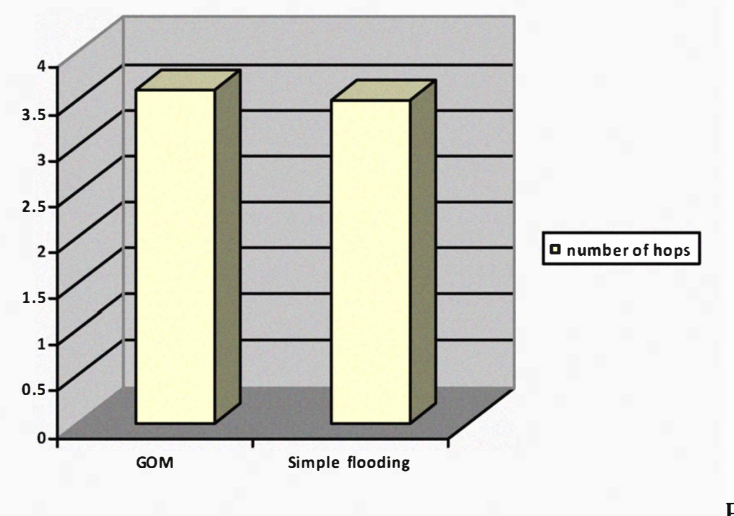

$\mathrm{Fi}$

Figure 5 Comparison based on number of hops

$$
\text { NHops }=\sum H
$$

Figure 5 shows the comparison of results of the two approaches (simple flooding and GOM) based on number of hops. We also comparing the redundancy between the two approaches with the formula (2) computing the redundancy where $N$ is number of nodes, $S$ is number of received messages in node $i$ and $D$ is current message. Figure 6 shows the comparison, where GOM outperforms simple flooding with respect to redundancy.

$$
\operatorname{Re} \text { dundancy }=\sum_{N}^{i=1} \sum_{S}^{m=2} D_{i m}
$$

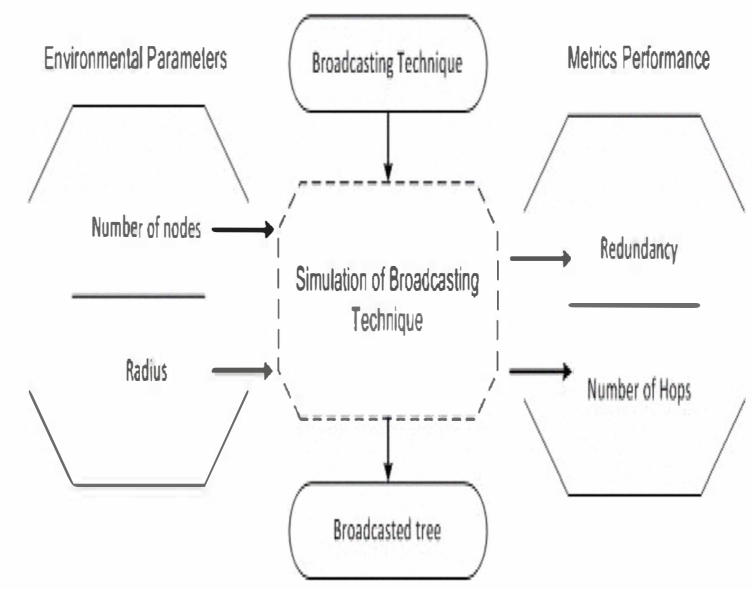

Figure 4 Block diagram of simulator 


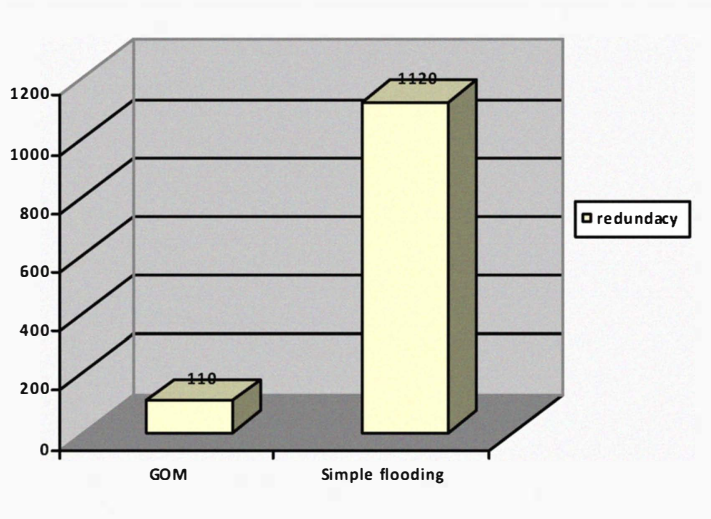

Figure 6 Comparison based on redundancy

\section{CONCLUSIONS AND FUTURE WORK}

In this paper, we have studied the storming problem faced while broadcasting data in a mobile ad-hoc network (MANET). We have proposed an optimised genetic algorithm to minimise the redundancy of message transmission MANETs. We have captured the parameters that change dynamically in such a wireless network so as to arrive at a fitness function for optimising the broadcasting tree by using genetic operations such as crossover, mutation and insertion effectively. The experimental results have clearly demonstrated the efficiency of our genetic optimisation model (GOM) as compared to simple flooding algorithm. The simulations show that while the number of hops is kept low and does not increase, the redundancy is reduced considerably (about 1:10 ratio), thereby effectively addressing the storming problem of MANETs. Our future work entails testing our GOM with other decision parameters such as latency.

\section{REFERENCES}

[1] Shaikh, J.; Solano, J.; Stojmenovic, I. \& Wu, J., " New metrics for dominating set based energy efficient activity scheduling in ad hoc networks", Proceedings of the 28th Annual IEEE International Conference on Local Computer Networks, 2003, 726

[2] Johnson, D.; Maltz, D.; Hu, Y. \& Jetcheva, J., "The dynamic source routing protocol for mobile ad hoc networks (DSR)", IETF Internet draft, 2003

[3] Tseng, Y.; Ni, S.; Chen, Y. \& Sheu, J., "The broadcast storm problem in a mobile ad hoc network", Wireless networks, Kluwer Academic Publishers, 2002, 8, 153-167.

[4] Al-Tabbakh, S.M., Amer, F.A., Belal, M.A. and Sakr, E.M., " A proposed distributed heuristic protocol for broadcasting in mobile ad hoc network", Egyptian Computer Science Journal, 2007, 29.

[5] Ho, C.; Obraczka, K.; Tsudik, G. \& Viswanath, K., "Flooding for reliable multicast in multi-hop ad hoc networks", Proceedings of the 3rd international workshop on Discrete algorithms and methods for mobile computing and communications, 1999, 71

[6] Jetcheva, J.; Hu, Y.; Maltz, D. \& Johnson, D., "A simple protocol for multicast and broadcast in mobile ad hoc networks", IETF MANET Working Group, Internet draft, 2001.

[7] Arango, J.; Degermark, M.; Efrat, A. \& Pink, S., "An efficient flooding algorithm for mobile ad-hoc networks", Proc. of WiOpt, 2004
[8] J. Lipman, P. Boustead, and J. Chicharo, "Reliable optimized flooding in ad hoc networks," in Proceedings of the IEEE 6th CAS Symposium on Emerging Technologies: Frontiers of Mobile and Wireless Communication, May 2004.

[9] Elaiwat, S. \& Belal, M., "An evolutionary creative design approach for optimising the broadcasting trees in MANET", International Journal of Design Engineering, Inderscience, 2010, 3, 97-114.

[10] Wu, B. \& Chao, K., "Spanning trees and optimization problems", CRC Press, 2004

[11] Ramosc, E. \& Skutellab, M., "Approximating k-hop minimumspanning trees", Operations Research Letters, Citeseer, 2005, 33, 115-120.

[12] Eppstein, D., "Spanning trees and spanners", Handbook of Computational Geometry, 2000, 425-461

[13] Garcia, S., Luque, C., Cervantes, A. \& Galvan, I.M. "Multiobjective Algorithms Hybridization to Optimize Broadcasting Parameters in Mobile Ad-Hoc Networks", In Cabestany, J. et al.(Eds.), LNCS, Springer, 2009, 728-735.

[14] Wu X., Wang, X. \& Liu, R., "Solving Minimum Power Broadcast Problem in Wireless Ad-Hoc Networks Using Genetic Algorithm", Communication Networks and Services Research Conference, 2008. CNSR 2008.

[15] Maalla, A., Wei, C. \& Taha, H.J., "Optimal Power Multicast Problem in Wireless Mesh Networks by Using a Hybrid Particle Swarm Optimization", American Journal of Applied Sciences, 6(9):, 2009, 1758-1762.

[16] Castro, M. C., Kassler, A. J., Chiasserini, C-F., Casetti, C. \& Korpeoglu, I., "Peer-to-Peer Overlay in Mobile Ad-hoc Networks", Handbook of Peer-to-Peer Networking, 2010, Part 9, 1045-1080. 